\title{
一氧化氮在 $\mathrm{Cu}_{3} P t(111)$ 表面的吸附行为
}

\author{
解秀娟 ${ }^{1}$ 陈文凯 ${ }^{1, *}$ 孙宝珍 $^{1}$ 郭 欣 ${ }^{2}$ 章永凡 ${ }^{1}$ \\ ( ${ }^{1}$ 福州大学化学系, 福州 350108; 2 华中科技大学煤燃烧国家重点实验室, 武汉 410074)
}

\begin{abstract}
摘要：运用密度泛函理论中广义梯度近似(GGA)的 PW91 方法, 结合周期性平板模型, 探讨了 NO 分子在 $\mathrm{Cu}_{3} \mathrm{Pt}(111)$ 表面上不同吸附位的吸附行为. 结果表明: $\mathrm{NO}$ 分子以 $\mathrm{N}$ 端朝下方式吸附在 top-Pt 以及 hepl 和 fcc2 位(分别为表面 $\mathrm{Cu}_{2} \mathrm{Pt}$ 和 $\mathrm{Cu}_{3}$ 簇)的吸附模式最稳定, 吸附能分别为 $101.8 、 124.5$ 和 $118.1 \mathrm{~kJ} \cdot \mathrm{mol}^{-1}$. 对于 hepl 和fcc2 位的吸附, $\mathrm{NO}$ 中的 $\mathrm{N}$ 原子分别与底物的 $\mathrm{Cu}_{2} \mathrm{Pt}$ 和 $\mathrm{Cu}_{3}$ 簇成键. 吸附前后的电荷布居、态密度和振动频率的分析 结果表明, 净电子从底物合金表面转移到 $\mathrm{NO}, \mathrm{N}-\mathrm{O}$ 键伸长, 频率发生红移. 合金 $\mathrm{Cu}_{3} \mathrm{Pt}$ 和纯贵金属 $\mathrm{Pt}$ 对 $\mathrm{NO}$ 的 吸附性质相似.
\end{abstract}

关键词：密度泛函理论；一氧化氮；铂铜合金；吸附 中图分类号: 0641

\section{Adsorption Behavior of NO on $\mathrm{Cu}_{3} \operatorname{Pt}(111)$ Surface}

\author{
XIE Xiu-Juan $^{1} \quad$ CHEN Wen-Kai ${ }^{1, *} \quad$ SUN Bao-Zhen ${ }^{1} \quad$ GUO Xin ${ }^{2} \quad$ ZHANG Yong-Fan ${ }^{1}$ \\ ('Department of Chemistry, Fuzhou University, Fuzhou 350108, P. R. China; ${ }^{2}$ State Key Laboratory of Coal Combustion, \\ Huazhong University of Science and Technology, Wuhan 410074, P. R. China)
}

\begin{abstract}
The adsorption behavior of $\mathrm{NO}$ onto a $\mathrm{Cu}_{3} \mathrm{Pt}$ (111) surface was studied using a periodic slab model and the PW91 generalized gradient approximation (GGA) within the framework of density functional theory. The calculated results indicated that $\mathrm{NO}$ adsorption with $\mathrm{N}$-down on the top-Pt, hcpl, and fcc2 sites resulted in favorable structures with predicted adsorption energies of $101.8,124.5$, and $118.1 \mathrm{~kJ} \cdot \mathrm{mol}^{-1}$, respectively. For adsorption onto the hcpl and fcc2 sites, $\mathrm{N}$ atom from $\mathrm{NO}$ formed bonds with $\mathrm{Cu}_{2} \mathrm{Pt}$ and $\mathrm{Cu}_{3}$ clusters, respectively. An analysis of the density of states, charge population, and vibrational frequencies before and after the adsorption showed that electrons transferred from the surface of the alloy to NO and that the $\mathrm{N}-\mathrm{O}$ bond was elongated and its vibrational frequency was redshifted. The $\mathrm{Cu}_{3} \mathrm{Pt}$ alloy and pure precious metal Pt have similar adsorption properties to NO.
\end{abstract}

Key Words: Density functional theory; $\mathrm{NO} ; \mathrm{Cu}_{3} \mathrm{Pt}$ alloy; Adsorption

工业领域中, 金属的合金化作为一种有效提高 表面反应活性的途径, 几十年来得到广泛的关注和 研究 ${ }^{[1-3]}$. 相对于纯金属, 合金表面的几何和电子结 构发生变化, 从而改变合金的选择和催化性能.一直 以来, $\mathrm{Pt}-\mathrm{Cu}$ 体系作为典型的二元合金体系得到广 泛研究 ${ }^{[4-6]}$. 通过探索 Pt-Cu 合金体系不同组分之间
的电子相互作用, 表明 Pt-Cu 合金体系作为烃重整 催化剂, 对石脑油脱氢裂解、 $\mathrm{CO}$ 的氧化、 $\mathrm{NO}_{x}$ 的储存 还原以及氢促进的氯代烃的脱氯反应起到催化作 用 ${ }^{[7]}$. Shen 等 ${ }^{[8]}$ 通过低能离子散射(LEIS)实验研究发 现 Pt-Cu 合金体系在高温条件下, 形成连续取代的 无规则结构. 但是, 在低温条件下三种有序相形成,

Received: July 12, 2010; Revised: August 28, 2010; Published on Web: September 30, 2010.

"Corresponding author. Email: qc2008@fzu.edu.cn; Tel: +86-591-22866162.

The project was supported by the National Natural Science Foundation of China (90922022), State Key Laboratory of Coal Combustion Foundation of Huazhong University of Science and Technology, China (FSKLCC0814), and New Century Excellent Talents Program in University of Fujian Province, China (HX2006-103, HX2006-98).

国家自然科学基金(90922022), 华中科技大学煤燃烧国家重点实验室基金(FSKLCC0814)和福建省高等学校新世纪优秀人才计划(HX2006-103, HX2006-98)资助项目 
分别为 $\mathrm{Cu}_{3} \mathrm{Pt}, \mathrm{CuPt}$ 和 $\mathrm{Pt}_{3} \mathrm{Cu}$. 此后, $\mathrm{Cu}_{3} \mathrm{Pt}$ 体系引起 研究者的极大兴趣. Shen 等昌对 $\mathrm{CO}$ 在 $\mathrm{Cu}_{3} \mathrm{Pt}(111)$ 表 面的吸附行为进行 LEIS 实验研究. 近年来 Zhang 等 ${ }^{[10]}$ 通过密度泛函理论(DFT)研究 $\mathrm{CO}$ 分子在 $\mathrm{Cu}_{3} \mathrm{Pt}$ (111)合金表面的氧化并与纯金属 $\mathrm{Pt}(111)$ 和 $\mathrm{Cu}(111)$ 表面进行比较. 研究表明, $\mathrm{CO}$ 在 $\mathrm{Cu}_{3} \mathrm{Pt}(111)$ 合金表 面的吸附能低于在两种纯金属表面的吸附能. 同时, $\mathrm{Cu}_{3} \mathrm{Pt}(111)$ 合金表面上 $\mathrm{CO}$ 氧化的势垒低于纯金属 表面, 推断 $\mathrm{Cu}_{3} \mathrm{Pt}$ 合金是一种比 $\mathrm{Pt}$ 和 $\mathrm{Cu}$ 更好的催 化剂. Linke ${ }^{[11]}$ 和 Dino ${ }^{[12]}$ 等分别从实验和理论方面研 究 $\mathrm{H}_{2}$ 在 $\mathrm{Cu}_{3} \mathrm{Pt}(111)$ 合金表面吸附和解离, 结果表明 $\mathrm{Cu}_{3} \mathrm{Pt}(111)$ 对 $\mathrm{H}_{2}$ 的解离有很好的催化作用. Basile 等 ${ }^{[13]}$ 运用 $X$ 射线衍射 $(X R D)$ 等实验方法研究 $\mathrm{Pt}-\mathrm{Cu} /$ 水滑石对 $\mathrm{NO}_{x}$ 的储存还原的催化作用, 研究表明 $\mathrm{Pt}-\mathrm{Cu}$ 合金的形成不仅使其催化性能较好, 且可以 防止催化剂的钝化. 金属 $\mathrm{Pt}$ 对 $\mathrm{NO}_{x}$ 的储存还原具 有很高的催化活性 ${ }^{[14-19]}$, 而且金属 $\mathrm{Cu}$ 是一种廉价金 属, 因此, Pt-Cu 合金对 $\mathrm{NO}_{x}$ 的储存还原催化性质的 研究显得十分重要. 因为大气中 $\mathrm{NO}_{x}$ (其中 $\mathrm{NO}$ 占 $90 \%$ 以上)的浓度随着近年来燃煤烟气和汽车尾气 的过度排放而呈现明显的上升趋势, 且 $\mathrm{NO}$ 在太阳 光作用下, 极易形成光化学烟雾. 目前对 NO 分子在 $\mathrm{Cu}_{3} \mathrm{Pt}$ 合金表面上的吸附分解的理论研究还未见报 道.

本文采用密度泛函理论结合周期平板模型方 法, 模拟 $\mathrm{NO}$ 分子在 $\mathrm{Cu}_{3} \mathrm{Pt}(111)$ 表面的吸附行为和 解离趋势, 对 $\mathrm{NO}$ 分子在 $\mathrm{Cu}_{3} \mathrm{Pt}(111)$ 表面不同吸附 位上的吸附构型进行了构型优化以及电荷布居、态 密度、轨道成分和振动频率的计算, 并讨论了不同吸
附位的稳定性和吸附能, 初步探讨 $\mathrm{NO}$ 分子与 $\mathrm{Cu}_{3} \mathrm{Pt}$ (111)表面相互作用的实质.

\section{1 计算模型和方法}

$\mathrm{Cu}_{3} \mathrm{Pt}$ 类似 $\mathrm{Cu}_{3} \mathrm{Au}$ 型, 属于面心立方堆积, $\mathrm{Pt}$ 原 子置于顶角位, $\mathrm{Cu}$ 原子置于晶胞的面心位 ${ }^{[20]}$. 在整 齐有序的 $\mathrm{Cu}_{3} \mathrm{Pt}(111)$ 表面, $\mathrm{Pt}$ 原子周围均为 $\mathrm{Cu}$ 原 子 ${ }^{[21-22]}$. 本文选取如图 1 所示的 $\mathrm{Cu}_{3} \mathrm{Pt}(111) 2 \times 2$ 超晶 胞四层平板模型研究 $\mathrm{NO}$ 分子在 $\mathrm{Cu}_{3} \mathrm{Pt}(111)$ 表面的 吸附.

本文采用广义梯度密度泛函理论和周期平板模 型方法模拟 $\mathrm{NO}$ 分子在 $\mathrm{Cu}_{3} \mathrm{Pt}(111)$ 表面的吸附. 所 有的计算均由 $\mathrm{Dmol}^{3}$ 软件包 ${ }^{[23-24]}$ 实现. 计算采用 PW91 泛函, $\mathrm{Pt}$ 和 $\mathrm{Cu}$ 原子内层电子由有效核电势 (ECP)代替, 价电子波函数采用双数值基加极化函数 (DNP) 展开, 非金属原子 $\mathrm{N}$ 和 $\mathrm{O}$ 采用全电子基组. Brillouin zone 积分的 Monkhorst-Pack 网格参数设 为 $3 \times 3 \times 1$, Methfessel-Paxton smearing 设为 $0.005 \mathrm{Ha}$; 结构优化以能量、位移和力收玫为判据, 收玫标准分 别为 $1 \times 10^{-5} \mathrm{Ha}, 5 \times 10^{-4} \mathrm{~nm}, 2 \times 10^{-4} \mathrm{Ha} \cdot \mathrm{nm}^{-1}$, 结构优 化时最底下三层原子固定在体相的位置, 上面一层 及 $\mathrm{N}$ 和 $\mathrm{O}$ 原子的坐标全部放开. 相邻两层平板间的 真空度厚度为 $1.3 \mathrm{~nm}$, 以确保平板间相互作用足够 小.

吸附能定义为吸附前后各物质总能量的变化:

$E_{\text {ads }}=E_{\text {adsorbate }}+E_{\text {substrate }}-E_{\text {adsorption system }}$

其中 $E_{\text {adsorbate }}$ 和 $E_{\text {substrate }}$ 分别表示吸附前吸附质和底物 的能量, $E_{\text {adsorption system }}$ 表示吸附后体系的总能量.

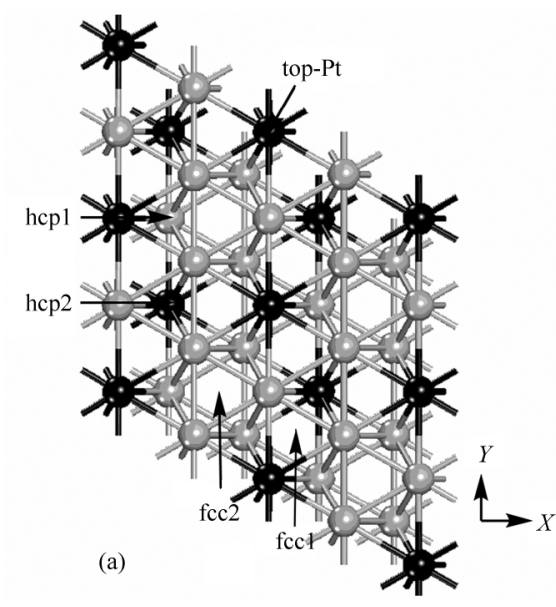

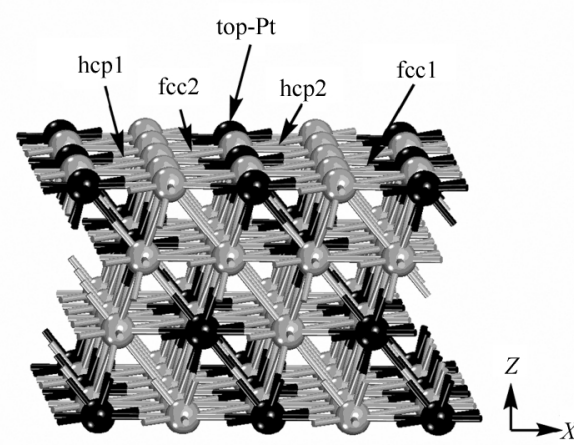

(b)

图 1 超晶胞模型的俯视图(a)和侧视图(b)

Fig.1 Top view (a) and side view (b) for the slab model Black and gray balls represent $\mathrm{Pt}$ atom and $\mathrm{Cu}$ atom, respectively. 


\section{2 结果与讨论}

\section{$2.1 \mathrm{NO}$ 分子在 $\mathrm{Cu}_{3} \mathrm{Pt}(111)$ 表面的吸附构型和吸} 附能

考虑 $\mathrm{NO}$ 在如图 1 所示 $\mathrm{Cu}_{3} \mathrm{Pt}(111)$ 表面的五种 不同吸附位, 分别是 top-Pt, fccl, fcc 2 , hcpl, hcp 2 , 其 中 1 代表 1 个 $\mathrm{Pt}$ 原子和 2 个 $\mathrm{Cu}$ 原子构成的穴位, 2 代表 3 个 $\mathrm{Cu}$ 原子构成的穴位. 在每个吸附位上, 考 虑 NO 分子三种初始吸附取向: $N$ 端朝下, $O$ 端朝下 和 $\mathrm{NO}$ 平行底物表面. 构型优化后, $\mathrm{NO}$ 分子以 $\mathrm{N}$ 端 朝下的吸附是化学吸附, $\mathrm{O}$ 端朝下为物理吸附, 平行 方式均转变为 $\mathrm{N}$ 端朝下. 因此, 为简明起见, 在 0.25 $\mathrm{Pt}$ 单层覆盖度下 $(0.25 \mathrm{ML})$, 所考察的三种化学稳定 吸附构型的构型参数和吸附能如图 2 所示.

由图 2 可见, 吸附能的大小顺序为 hcpl $>f \mathrm{fc} 2>$ top-Pt, 吸附能愈大, 吸附分子与衬底之间的吸附愈 强, 由此可见 NO 以 $\mathrm{N}$ 端在 hepl 位的吸附最强. 值 得指出的是, $\mathrm{NO}$ 在 hcpl 位和 fcc2 位及其 top-Pt 位 的吸附能差值不是非常大, 因此 NO 以 $\mathrm{N}$ 端吸附在 这三种位置的吸附模式在能量上比较有利, 均为稳 定的吸附构型.

对于 $\mathrm{N}-\mathrm{O}$ 键, $\mathrm{NO}$ 在 $\mathrm{Cu}_{3} \mathrm{Pt}(111)$ 表面的这 3 个 吸附位的吸附均使 $\mathrm{N}-\mathrm{O}$ 键相对于自由 $\mathrm{NO}$ 分子 (计算值为 $0.1163 \mathrm{~nm}$, 实验值为 $0.1151 \mathrm{~nm}^{[25]}$ ) 伸长, 但 top-Pt 位上 $\mathrm{N}$ - $\mathrm{O}$ 键长变化并不明显, 而在 hcpl 和 $\mathrm{fcc} 2$ 位 $\mathrm{N}-\mathrm{O}$ 键长变化非常显著, 而且 $\mathrm{N}-\mathrm{O}$ 键
表 1 NO 分子在 $\mathrm{Cu}_{3} \operatorname{Pt}(111)$ 和 $\operatorname{Pt}(111)$ 不同吸附位的振动 频率

Table 1 Vibrational frequencies of NO on different adsorptional sites for $\mathrm{Cu}_{3} \mathrm{Pt}(\mathbf{1 1 1})$ and $\mathrm{Pt}(111)$ surfaces

\begin{tabular}{cccc}
\hline Adsorption model & $\nu_{\mathrm{No}} / \mathrm{cm}^{-1}$ & $\nu_{\mathrm{NO}}(\text { theo })^{\mathrm{b}} / \mathrm{cm}^{-1}$ & $\nu_{\mathrm{NO}}(\exp )^{\mathrm{c}} / \mathrm{cm}^{-1}$ \\
\hline top-Pt & 1761 & 1702 & 1715 \\
hcpl & 1498 & 1529 & 1508 \\
fcc2 & 1463 & 1502 & 1490 \\
free NO & $1884^{\mathrm{a}}$ & 1899 & 1876 \\
\hline
\end{tabular}

${ }^{\mathrm{a}}$ predicted value; ${ }^{\mathrm{b}}$ the theoretical values in Ref.[18] for NO molecules on $\mathrm{Pt}(111)$ surface; ${ }^{\mathrm{c}}$ the experimental values in Ref.[27] for NO molecules on $\mathrm{Pt}(111)$ surface

长均在 $(0.118 \pm 0.004) \mathrm{nm}$ 范围, 这与实验测得 $\mathrm{NO}$ 在 纯金属 $\mathrm{Pt}(111)$ 的吸附键长范围 ${ }^{[26]}$ 相吻合. 可见 $\mathrm{NO}$ 在 $\mathrm{Cu}_{3} \operatorname{Pt}(111)$ 表面的吸附和在 $\operatorname{Pt}(111)$ 表面的吸附 同样造成了 $\mathrm{NO}$ 的 $\mathrm{N}-\mathrm{O}$ 键削弱, 有利于 $\mathrm{NO}$ 的进 一步解离. 文献 ${ }^{[10]}$ 中, $\mathrm{CO}$ 稳定吸附在 $\mathrm{Cu}_{3} \mathrm{Pt}(111)$ 表 面 top-Pt 位的吸附能为 $1.51 \mathrm{eV}\left(145.7 \mathrm{~kJ} \cdot \mathrm{mol}^{-1}\right)$, 其 大于本文 $\mathrm{NO}$ 在 $\mathrm{Cu}_{3} \mathrm{Pt}(111)$ 表面 top-Pt 位的吸附能 $\left(101.8 \mathrm{~kJ} \cdot \mathrm{mol}^{-1}\right)$, 但根据 $\mathrm{N}-\mathrm{O}$ 和 $\mathrm{C}-\mathrm{O}$ 键的活化难 易程度, $\mathrm{N}-\mathrm{O}$ 键可能更容易, 这是因为 $\mathrm{NO}$ 分子的 解离能远低于 $\mathrm{CO}$ 分子的解离能.

\section{$2.2 \mathrm{NO}$ 的伸缩振动频率分析}

表 1 为 $\mathrm{NO}$ 在 $\mathrm{Cu}_{3} \mathrm{Pt}(111)$ 表面不同吸附位的振 动频率和 $\mathrm{NO}$ 在 $\mathrm{Pt}(111)$ 表面的理论 ${ }^{[18]}$ 及实验 ${ }^{[27]}$ 振 动频率值. 由表可见, 我们计算得出自由分子 $\nu_{\mathrm{NO}}$ 为 $1884 \mathrm{~cm}^{-1}$, 与实验值 $1876 \mathrm{~cm}^{-1}$ 相符合. 对于 $\mathrm{Cu}_{3} \mathrm{Pt}$

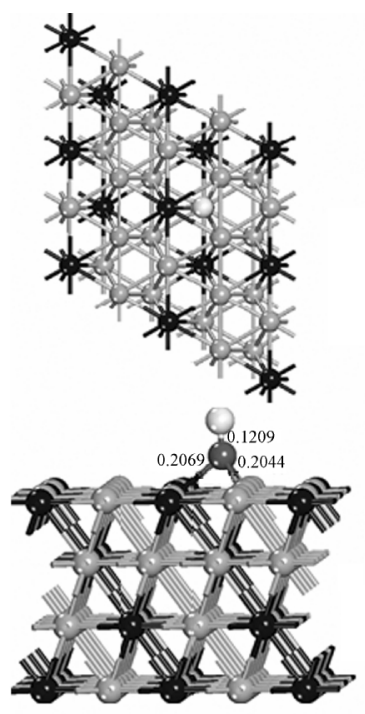

$E_{\text {ads }}=124.5 \mathrm{~kJ} \cdot \mathrm{mol}^{-1}$

(a)

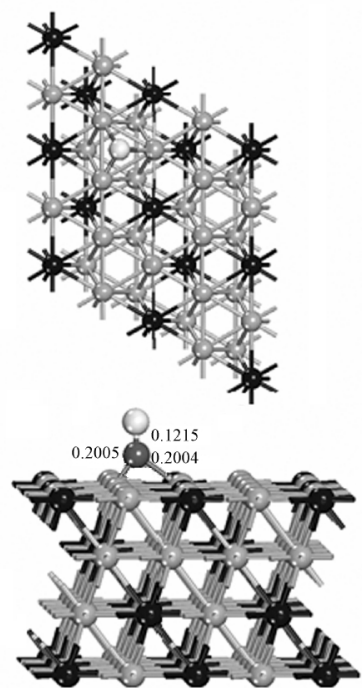

$E_{\mathrm{ads}}=118.1 \mathrm{~kJ} \cdot \mathrm{mol}^{-1}$

(b)

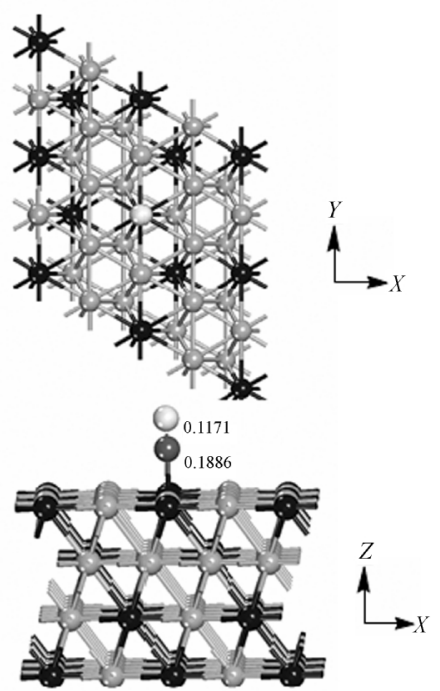

$E_{\mathrm{ads}}=101.8 \mathrm{~kJ} \cdot \mathrm{mol}^{-1}$

(c)

图 $2 \mathrm{NO}$ 分子在 $\mathrm{Cu}_{3} \mathrm{Pt}(111)$ 表面的三种稳定吸附构型

Fig.2 Three stable structures of NO adsorbed on $\mathrm{Cu}_{3} \mathrm{Pt}(111)$ surface (a) hcpl site; (b) fcc2 site; (c) top-Pt site 
表 $2 \mathrm{NO}$ 中各原子和 $\mathrm{Cu}$ 及 $\mathrm{Pt}$ 原子的平均 Mulliken 电荷布居

Table 2 Average Mulliken populations for $N$, 0 in $\mathrm{NO}$ and $\mathrm{Cu}, \mathrm{P}$ atoms

\begin{tabular}{cccccc}
\hline Adsorption model & $q_{\mathrm{N}}$ (a.u.) & $q_{\mathrm{o}}$ (a.u.) & $q_{\mathrm{NO}}($ a.u. $)$ & $q_{\mathrm{Pt}}($ a.u. $)$ & $q_{\mathrm{cu}}($ a.u. $)$ \\
\hline top-Pt & 0.184 & -0.015 & 0.169 & -0.293 & - \\
hcp1 & 0.046 & -0.096 & -0.050 & -0.288 & $0.119 / 0.120$ \\
fcc2 & 0.076 & -0.104 & -0.028 & - & $0.112 / 0.112 / 0.112$ \\
free $^{\mathrm{a}}$ & 0.040 & -0.040 & 0.000 & -0.406 & 0.075 \\
\hline \multicolumn{5}{c}{${ }^{\mathrm{a}}$ predicted value }
\end{tabular}

(111)表面, NO 吸附后, 频率红移, 尤其在 hcpl和 fcc2 稳定吸附位, 红移了 $400 \mathrm{~cm}^{-1}$ 左右, 这与 $\mathrm{N}-\mathrm{O}$ 键长 伸长, 键被削弱的结果相符合. 而且 NO频率红移的 大小与键长的伸长程度及吸附能大小的变化趋势一 致. Getman 等 ${ }^{[18]}$ 用 DFT 研究 NO 在 Pt(111) 表面的 吸附, 计算得出的 top, hep 和 fcc 吸附位 NO 的振动 频率的变化趋势与我们的结果一致, 并且均与实验 值一致. 以上事实进一步说明 $\mathrm{Cu}_{3} \mathrm{Pt}(111)$ 表面对 $\mathrm{NO}$ 的吸附与纯贵金属 $\operatorname{Pt}(111)$ 表面的吸附性质相近. 从 而我们推测 $\mathrm{Cu}_{3} \mathrm{Pt}$ 合金和纯贵金属 $\mathrm{Pt}$ 对 $\mathrm{NO}$ 的催化 性能相似。

\subsection{Mulliken 电荷布居和轨道布居分析}

表 2 给出了吸附前后 $\mathrm{NO}$ 各原子和底物金属原 子的 Mulliken 电荷布居, 由表 2 可见, 吸附后, NO 既是得电子体, 又是失电子体. 因为 $\mathrm{NO}$ 分子的电子 组态为: $\mathrm{NO}\left[(1 \sigma)^{2}(2 \sigma)^{2}(3 \sigma)^{2}(4 \sigma)^{2}(1 \pi)^{4}(5 \sigma)^{2}(2 \pi)^{1}\right]^{[28]}$, 即最高占据轨道(HOMO) 和最低未占据轨道(LUMO) 均为半充满的 $2 \pi$ 反键轨道. 这意味着当 $\mathrm{NO}$ 分子 吸附在固体表面时, 它既可以从表面接受电子, 又可 以将电子反馈到固体表面. 在 hcpl 和 fcc2 两个吸 附位, $\mathrm{NO}$ 均得到电子, $\mathrm{Pt}$ 和 $\mathrm{Cu}$ 原子均失去电子. 值 得注意的是, 在 top-Pt 吸附位, NO 分子和 Pt 原子均 失去电子, 为了解释这个看似矛盾的结论, 表 3 给出 $\mathrm{NO}$ 吸附前后的轨道布居. 由表可知, 与自由 NO 分 子相比, 处于 top-Pt 位吸附时 $\mathrm{N}$ 原子和 $\mathrm{O}$ 原子的 $2 p_{x}, 2 p_{y}$ 轨道均得到电子, 根据我们实验组之前的研 究 ${ }^{[29]}$ 表明, $\mathrm{NO}$ 分子的 $2 \pi$ 反键轨道是由 $\mathrm{N}$ 和 $\mathrm{O}$ 原

表 $3 \mathrm{~N}$ 和 $\mathrm{O}$ 原子在 top-Pt 位吸附前后的轨道布居

Table 3 Orbital populations for $\mathrm{N}$ and $\mathrm{O}$ atoms on top-Pt site before and after adsorption

\begin{tabular}{|c|c|c|c|c|c|c|c|c|}
\hline \multirow{2}{*}{ Orbital } & \multicolumn{4}{|c|}{$\mathrm{N}$ atom } & \multicolumn{4}{|c|}{$\mathrm{O}$ atom } \\
\hline & $2 s$ & $2 p_{x}$ & $2 p_{y}$ & $2 p_{z}$ & $2 s$ & $2 p_{x}$ & $2 p_{y}$ & $2 p_{z}$ \\
\hline free & 1.851 & 1.005 & 1.005 & 1.022 & 1.818 & 1.449 & 1.449 & 1.263 \\
\hline top-Pt & 1.607 & 1.130 & 1.130 & 0.838 & 1.812 & 1.473 & 1.473 & 1.198 \\
\hline$\Delta$ & -0.244 & 0.125 & 0.125 & -0.184 & -0.006 & 0.024 & 0.024 & 0.065 \\
\hline
\end{tabular}

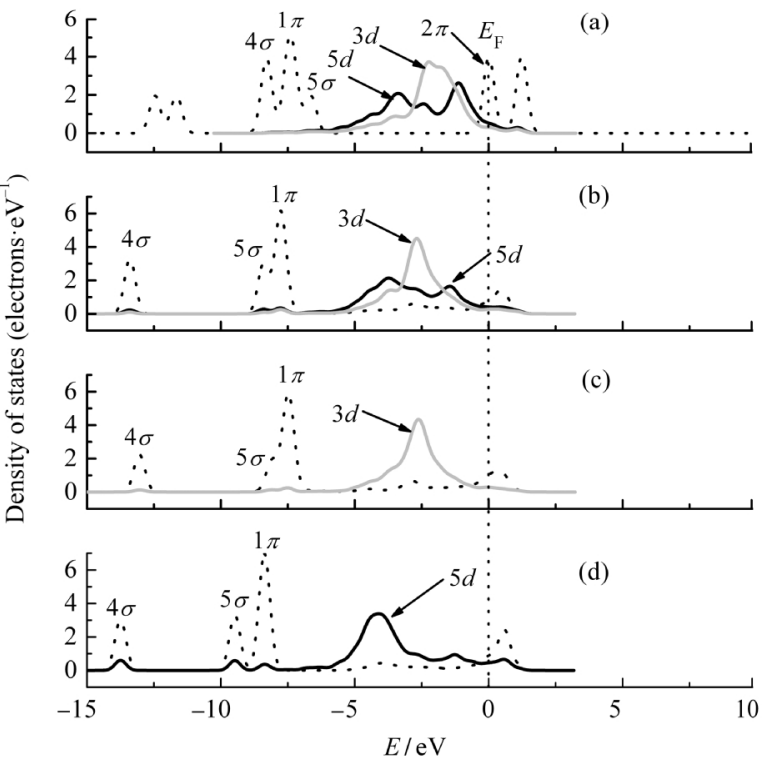

图 3 吸附前后 NO 分子和吸附位 Pt 及 $\mathrm{Cu}$ 原子 态密度的变化

Fig.3 Density of states for NO and the $\mathrm{Pt}$ and $\mathrm{Cu}$ atoms on the adsorption sites before and after NO adsorption on $\mathrm{Cu}_{3} \mathrm{Pt}(111)$ surface

(a) before adsorption, (b) hcpl after adsorption, (c) fccl after adsorption, (d) top-Pt after adsorption; $\cdots \mathrm{NO}, \longrightarrow \mathrm{Cu}, \longrightarrow \mathrm{Pt}$

子的 $2 p$ 轨道杂化形成. 由此可以得出 $\mathrm{NO}$ 分子在 top-Pt 位吸附后其 $2 \pi$ 反键轨道得到电子, 导致键级 变小, $\mathrm{N}-\mathrm{O}$ 键长伸长. 但是在 Getman ${ }^{\text {等 }}{ }^{[18]}$ 的文献 报道中, $\mathrm{NO}$ 在 $\mathrm{Pt}(111)$ 表面的 top, hcp 及 $\mathrm{fcc}$ 位的吸 附, 均为得到电子. 说明 $\mathrm{Cu}$ 和 $\mathrm{Pt}$ 合金化在一定程度 上改变了 $\mathrm{NO}$ 电子的转移方式.

\section{4 态密度分析}

图 3 给出了吸附前后 NO 分子和吸附位 Pt 及 $\mathrm{Cu}$ 原子的态密度图. 与吸附前的态密度相比, 吸附 后的的 NO 分子的 $2 \pi$ 反键轨道的态密度峰发生了 明显的离域化, 与 $\mathrm{Cu} 3 d$ 和 $\mathrm{Pt} 5 d$ 轨道的态密度峰发 生明显重叠, 说明 $\mathrm{NO}$ 分子在吸附后与 $\mathrm{Cu}$ 及 $\mathrm{Pt}$ 原 子形成化学键, 且造成 $\mathrm{N}-\mathrm{O}$ 键长伸长, 频率红移, 这与我们前面分析的相符合. NO 分子发生红移是 由于电子流从 $\mathrm{Cu}_{3} \mathrm{Pt}$ 表面转移到吸附分子所导致, 即 NO 分子得到电子, 这与上述 Mulliken 电荷分析 结果相一致. 在文献 ${ }^{[10]}$ 中, 通过对比纯金属 $\mathrm{Pt}$ 和合 金 $\mathrm{Cu}_{3} \mathrm{Pt}$ 中 $\mathrm{Pt}$ 原子的态密度, 表明合金化之后, $\mathrm{Pt}$ 原子的带中心向低能级移动了 $0.12 \mathrm{eV}$. 这就稍微降 低了 $\mathrm{Pt}$ 的 $d$ 轨道与 $\mathrm{NO}$ 的 $2 \pi$ 反键轨道的成键作 用, 即 $\mathrm{NO}$ 在合金 $\mathrm{Cu}_{3} \mathrm{Pt}$ 上的吸附会稍弱于在纯金 属 Pt 上的吸附. 


\section{3 结 论}

采用密度泛函理论, 模拟了 $\mathrm{NO}$ 分子吸附在 $\mathrm{Cu}_{3} \mathrm{Pt}(111)$ 表面的平衡几何结构和相应的电子结构. 对 $\mathrm{NO}$ 分子在 $\mathrm{Cu}_{3} \mathrm{Pt}(111)$ 表面不同吸附位上的吸附 进行了构型优化. 结果表明, $\mathrm{NO}$ 分子以 $\mathrm{N}$ 端朝下方 式吸附在 top-Pt, hcpl 和 fcc 2 位最稳定, 且 $\mathrm{N}-\mathrm{O}$ 键 伸长, 有进一步解离的可能. Mulliken 电荷布居分析 结果表明, 吸附过程中 NO 既是得电子体又是失电 子体. 振动频率的结果表明, 吸附后 NO 频率发生红 移, 进一步说明 $\mathrm{N}-\mathrm{O}$ 键的削弱. 并且通过与文献报 道中 $\mathrm{NO}$ 在 $\mathrm{Pt}(111)$ 表面吸附的比较, 表明 $\mathrm{Cu}_{3} \mathrm{Pt}$ 合金 和纯贵金属 Pt 对 NO 的吸附性质相似, 进一步推测 它们对 NO 的催化性能也可能相似. 当然, 这还取决 于 NO 后面的解离、成键和脱附行为.

\section{References}

1 Ponec, V. Catal. Rev., 1975, 11: 41

2 Campbell, C. Annu. Rev. Phys. Chem., 1990, 41: 775

3 Bardi, U. Rep. Prog. Phys., 1994, 57: 939

4 Knudsen, J.; Nilekar, A. U.; Vang, R. T.; Schnadt, J.; Kunkes, E. L.; Dumesic, J. A.; Mavrikakis, M.; Besenbacher, F. J. Am. Chem. Soc., 2007, 129: 6485

5 Luyten, J.; Schurmans, M.; Creemers, C.; Bunnik, B. S.; Kramer, G. J. Surf. Sci., 2007, 601: 2952

6 Pasti, I.; Mentus, S. Mater. Chem. Phys., 2009, 116: 94

7 Avdeev, V. I.; Kovalchuk, V. I.; Zhidomirov, G. M.; d'Itri, J. L. Surf. Sci., 2005, 583: 46

8 Shen, Y. G.; O'Connor, D. J.; Wandelt, K.; MacDonald, R. J. Surf. Sci., 1995, 328: 21

9 Shen, Y. G.; O'Connor, D. J.; Wandelt, K.; MacDonald, R. J. Surf. Sci., 1995, 331-333: 746

10 Zhang, C. J.; Baxter, R. J.; Hu, P.; Alavi, A.; Lee, M. H. J. Chem Phys., 2001, 115: 5272
11 Linke, R.; Schneider, U.; Busse, H.; Becker, C.; Schröder, U.; Castro, G. R.; Wandelt, K. Surf. Sci., 1994, 307-309: 407

12 Dino, W. A.; Kasai, H.; Okiji, A. Surf. Sci., 2001, 482-485: 318

13 Basile, F.; Fornasari, G.; Livi, M.; Tinti, F.; Trifiro, F.; Vaccari, A. Top. Catal., 2004, 30/31: 223

14 Abe, A.; Yamashita, K. Chem. Phys. Lett., 2004, 393: 331

15 Zhu, P.; Shimada, T.; Kondoh, H.; Nakai, I.; Nagasaka, M.; Ohta, T. Surf. Sci., 2004, 565: 232

16 Jiang, Z.; Huang, W.; Tan, D.; Zhai, R.; Bao, X. Surf. Sci., 2006, 600: 4860

17 Tsukahara, N.; Mukai, K.; Yamashita, Y.; Yoshinobu, J.; Aizawa, H. Surf. Sci., 2006, 600: 3477

18 Getman, R. B.; Schneider, W. F. J. Phys. Chem. C, 2007, 111: 389

19 Smeltz, A. D.; Getman, R. B.; Schneider, W. F.; Ribeiro, F. H. Catal. Today, 2008, 136: 84

20 Hansen, M.; Anderko, K. Constitution of binary alloys. 2nd ed. New York: McGraw-Hill, 1958

21 Castro, G. R.; Schneider, U.; Busse, H.; Janssens, T.; Wandelt, K. Surf. Sci., 1992, 269-270: 321

22 Schneider, U.; Castro, G. R.; Busse, H.; Janssens, T.; Wesemann, J.; Wandelt, K. Surf. Sci., 1992, 269-270: 316

23 Delley, B. J. Chem. Phys., 1990, 92: 508

24 Delley, B. J. Chem. Phys., 2000, 113: 7756

25 Lide, D. CRC handbook of chemistry and physics. Boca Raton: CRC Press, 2003: 9-20

26 Materer, N.; Barbieri, A.; Gardin, D.; Starke, U.; Batteas, J. D.; Vanhove, M. A.; Somorjai, G. A. Physical Review B, 1993, 48 2859

27 Matsumoto, M.; Tatsumi, N.; Fukutani, K.; Okano, T. Surf. Sci., 2002, 513: 485

28 Pan, D. A.; Zhao, C. D.; Zheng, Z. X. Structures of matters. 2nd ed Beijing: Higher Education Press, 1989: 200-201［潘道皑, 赵成 大, 郑载兴. 物质结构. 北京: 高等教育出版社, 1989: 200-201]

29 Sun, B. Z.; Chen, W. K.; Zheng, J. D.; Lu, C. H. Appl. Surf. Sci., 2008, 255: 3141 